Agronomía Costarricense 43(1): 35-45. ISSN:0377-9424 / 2019

www.mag.go.cr/rev_agr/index.html www.cia.ucr.ac.cr

\title{
COMPARACIÓN DE DOS DISOLUCIONES EXTRACTORAS DE BORO Y DETERMINACIÓN DE SU CONTENIDO EN EL SUELO Y EN LA PLANTA DE BANANO
}

\author{
Veda Obando-Bustos ${ }^{1 *}$, Rafael Segura-Mena*, Jorge Sandoval-Fernández* \\ Palabras clave: Fosfato de calcio; Mehlich 3; microelementos; Musa AAA; subgrupo Cavendish. \\ Keywords: Calcium phosphate; Mehlich 3; microelements; Musa AAA; Cavendish subgroup.
}

Recibido: 22/01/18

Aceptado: $30 / 07 / 18$

\section{RESUMEN}

El objetivo del estudio fue comparar 2 disoluciones extractoras de boro (fosfato de calcio y Mehlich 3), y determinar su contenido en el suelo y en la planta de banano (tercera hoja). El trabajo se efectuó en el Laboratorio Químico de CORBANA S.A., ubicado en la provincia de Limón, cantón de Pococí, distrito La Rita, en el 2015. Para tal propósito se colectaron muestras de suelo y de tejido foliar provenientes de plantaciones bananeras situadas en el Caribe costarricense. Suelos con contenidos inferiores a $0,2 \mathrm{mg} . \mathrm{l}^{-1} \mathrm{de}$ boro presentaron una mayor extracción del elemento con la disolución Mehlich 3, en comparación con la disolución de fosfato de calcio. Contenidos entre 0,2 y $0,7 \mathrm{mg} . \mathrm{l}^{-1}$ mostraron, por su parte, una correlación del $68 \%$ entre ambas disoluciones. Niveles superiores a 2 mg. ${ }^{-1}$ exhibieron una correlación del $98 \%$. El noventa por ciento de las muestras de suelo analizadas presentaron contenidos de boro menores a 1,0 mg. $l^{-1}$, con la mayoría de las muestras $(64 \%)$ en concentraciones de 0,2 a $0,7 \mathrm{mg} \cdot \mathrm{l}^{-1}$. El ámbito de boro recomendado para la planta de banano es de 0,1 a $1,0 \mathrm{mg} \cdot \mathrm{l}^{-1}$, por lo que la mayoría de los suelos muestreados presentaron valores adecuados.

1 Autora para correspondencia. Correo electrónico: vobando@corbana.co.cr

\section{ABSTRACT}

Comparation of two boron extractant solutions and determination of its content in soil and banana plant. The objective of the study was to compare 2 boron extracting solutions (calcium phosphate and Mehlich 3), as well as to determine its content in soil and banana plant (third leaf). The study was carried out in the Chemical Laboratory of CORBANA SA, located in the province of Limón, Pococí county, La Rita district, in 2015. For this purpose, soil samples and leaf tissue were collected from banana plantations located in the Caribbean of Costa Rica. Soils with boron concentration lower than $0.2 \mathrm{mg} . \mathrm{l}^{-1}$ had a greater extraction of the element with the Mehlich 3 solution, in comparison with the calcium phosphate solution. Content between 0.2 and $0.7 \mathrm{mg} . \mathrm{l}^{-1}$ showed, on the other hand, a correlation of $68 \%$ between both solutions. Concentrations higher than 2 mg. $\mathrm{l}^{-1}$ exhibited a correlation of $98 \%$. Ninety percent of analyzed soil samples had boron concentrations of less than $1.0 \mathrm{mg} . \mathrm{l}^{-1}$, with the most samples (64\%) at contents of 0.2 to $0.7 \mathrm{mg} . \mathrm{l}^{-1}$. Recommended boron concentration for banana plants should range between 0.1 to $1.0 \mathrm{mg} . \mathrm{l}^{-1}$, so most sampled soils * Corporación Bananera Nacional (CORBANA),
Costa Rica. 
Las concentraciones foliares de boro fueron de 10 a 15 mg.kg- en la sección central interna de la tercera hoja, mientras que en la sección externa de la hoja (borde) fueron de 10 a 70 mg. $\mathrm{kg}^{-1}$ con un aumento de su concentración hacia el borde de la hoja. Los resultados indican la bondad del uso de la disolución de Mehlich 3 sobre la disolución de fosfato de calcio para la determinación de boro en muestras de suelo.

\section{INTRODUCCIÓN}

La información sobre metodologías de extracción de boro en suelo mediante el uso de diferentes disoluciones extractoras es numerosa (Redd et al. 2008, Moreira et al. 2011). Entre estas se encuentra la extracción del elemento con agua caliente (Berger y Troug 1939), las disoluciones Mehlich 3 y Mehlich 1 (Mehlich 1984, Schuman et al. 1992), acetato de amonio (Matula 2009), cloruro de calcio (Jeffrey y McCallum 1988) y cloruro de potasio (Moreira et al. 2011). La extracción con agua caliente se ha considerado como el método de referencia, sin embargo, presenta una alta variabilidad y es más laborioso (Allen et al. 2005, Redd et al. 2008).

Diversos autores (Schuman et al. 1992, Matula 2009, Zbíral 2016) indican que la disolución extractora Mehlich 3 se correlaciona estrechamente con el contenido de boro en el suelo, producto de aplicaciones diferenciadas del microelemento.

En Costa Rica y para el cultivo del banano, la Corporación Bananera Nacional (CORBANA S.A.), por medio de su Laboratorio Químico, realizó por varios años la determinación de boro en suelo mediante una disolución extractora de fosfato de calcio $\left(\mathrm{CaH}_{4}\left(\mathrm{PO}_{4}\right)_{2}, 0,008 \mathrm{M}\right)$ (Romeu-Díaz y Hunter 1978). Este procedimiento tenía el inconveniente de ser diferente al procedimiento de extracción (Mehlich 3) para los presented adequate values. Foliar boron contents were 10 to $15 \mathrm{mg} \cdot \mathrm{kg}^{-1}$ in the inner central section of the third leaf, while in the outer section of the leaf (border) they were 10 to $70 \mathrm{mg} \cdot \mathrm{kg}^{-1}$ with an increase in its concentration towards the edge of the leaf. Results indicate the advantage of using the Mehlich 3 over the calcium phosphate solution for the determination of boron in soil samples.

demás elementos y, además, su utilización era esporádica. Ante tal perspectiva, para uniformar el procedimiento general de análisis de suelo para los demás elementos que se determinan en CORBANA, se consideró evaluar la extracción de boro con Mehlich 3 .

El boro es reconocido como un micronutriente esencial para las plantas, está involucrado en el transporte de azúcares a través de las membranas celulares, la síntesis e integridad estructural de la pared celular, el desarrollo y crecimiento de procesos reproductivos, el metabolismo de las plantas, así como en la estructura $\mathrm{y}$ funcionamiento de la membrana celular (Brown et al. 2002).

El contenido total de boro en el suelo varía de 2 a 200 mg.kg-1 ${ }^{-1}$ sin embargo, sólo una pequeña fracción (3 a 5\%) está disponible para los cultivos. El boro disponible por lo general es menor a 5 mg. $\mathrm{kg}^{-1}$ (Arunkumar et al. 2018).

Vargas y Solís (1999) encontraron que la deficiencia de boro en plantas de banano Musa $A A A$, en condiciones hidropónicas, se localizó en las hojas más jóvenes con un gran número de pequeñas líneas translúcidas distribuidas por toda la lámina foliar y paralelas a la vena central. La hoja se deformó y presentó un corrugamiento marginal. Por el contrario, el exceso de boro en la planta de banano en condiciones hidropónicas mostró una decoloración marginal de la hoja y 
con necrosis marginal delgada, discontinua e irregular (Vargas 1999).

Un suplemento excesivo de boro crea un gradiente de su contenido, que aumenta del peciolo a la lámina foliar y de esta a la punta de la hoja. Por consiguiente, la necrosis de los márgenes o de las puntas de las hojas, se produce como consecuencia de dicha condición (Marschner 1995). La toxicidad de boro puede presentarse con valores cercanos a $30 \mathrm{mg} \cdot \mathrm{kg}^{-1}$ (Vargas et al. 2007).

$\mathrm{La}$ aparición de lesiones foliares en la plantas de banano (Musa AAA) en áreas comerciales de cultivo intensivo plantea interrogantes sobre el origen de las mismas. Por otro lado, la determinación del contenido de boro en suelos ha representado un desafío para los laboratorios de análisis químico. Ello por la restringida utilidad del análisis, debido al amplio porcentaje de variación de los resultados, que podía ser de hasta un 50 o $60 \%$ (Corrales et al. 2005).

El objetivo del estudio fue comparar la disolución extractora Mehlich 3 contra la disolución de fosfato de calcio, así como determinar el contenido de boro en el suelo de fincas bananeras y en la planta de banano (tercera hoja).

\section{MATERIALES Y MÉTODOS}

El estudio se efectuó en las instalaciones del Laboratorio Químico de CORBANA S.A., ubicado en la provincia de Limón, cantón de Pococí, distrito La Rita, durante el 2015. Este consistió en la evaluación de: 1-disoluciones extractoras Mehlich 3 y fosfato de calcio, 2-contenido de boro en suelos de fincas bananeras y 3 -contenido de boro en la planta de banano (tercera hoja).

\section{Disoluciones extractoras Mehlich 3 $y$ fosfato de calcio}

Se tomaron 295 muestras de suelo provenientes de fincas bananeras localizadas en el Caribe de Costa Rica. Las muestras de suelo fueron tomadas en la banda de fertilización, frente al hijo de sucesión, a una profundidad de 10-30 cm, secadas a $40^{\circ} \mathrm{C}$ por 3 días y molidas a un tamaño de partícula menor a $2 \mathrm{~mm}$. Para la extracción con fosfato de calcio se midieron $10 \mathrm{ml}$ de muestra, se agregó $25 \mathrm{ml}$ de disolución extractora y se agitó por 10 minutos a $200 \mathrm{rpm}$ en un agitador oscilatorio para luego filtrar la muestra. Para la extracción con Mehlich 3, se midieron 2,5 ml de muestra; se agregó $10 \mathrm{ml}$ de disolución extractora y se agitó por 5 minutos a 200 rpm en un agitador oscilatorio. Después de 5 minutos de reposo se filtró. Para ambas disoluciones extractoras el líquido resultante fue analizado en un equipo ICP/AES PerkinElmer Optima 7300 DV $®$ a una longitud de onda de 249,676 nm.

Para la preparación de la disolución Mehlich 3 y los estándares de calibración, se utilizaron recipientes de plástico (polipropileno), debido a que esta disolución incluye fluoruro de amonio $\left(\mathrm{NH}_{4} \mathrm{~F}\right)$ que reacciona con la cristalería de vidrio borosilicato (Allen et al. 2005).

Adicionalmente, para el análisis de boro en el suelo, se llevó a cabo un ajuste conocido como MSF (Multicomponent Spectral Fitting) en la lectura del equipo de plasma ICP-OES para eliminar la interferencia de hierro en la lectura de boro y mejorar el límite de detección del método (PerkinElmer 1996).

Se analizaron muestras de referencia del programa International Soil Analytical-Exchange (ISE) de Wageningen Evaluating Programs for Analytical Laboratories de Holanda (WEPAL) con diferentes contenidos de boro, con el fin de confirmar si la metodología genera resultados comparables con otros laboratorios en el mundo y, de esa forma, asegurar la exactitud (WEPAL 2015a).

\section{Contenido de boro en suelos de fincas bananeras}

Se tomaron 319 muestras de suelo adicionales a las 295 muestras ya mencionadas, también provenientes de fincas bananeras localizadas en el Caribe de Costa Rica, muestreadas en la banda de fertilización, frente al hijo de sucesión, a una profundidad de $10-30 \mathrm{~cm}$, secadas a $40^{\circ} \mathrm{C}$ por 3 días y molidas a un tamaño de partícula menor a $2 \mathrm{~mm}$. Estas muestras fueron extraídas con la disolución Mehlich 3 y leídas en el equipo de plasma ICP/OES (PE Optima 7300 DV) para la determinación y estudio de boro. 
Los resultados referentes al contenido de boro en el suelo de fincas bananeras consideraron las 614 muestras totales.

\section{Contenido de boro en la planta de banano (tercera hoja)}

Se determinó el contenido de boro foliar en 87 muestras de plantas de banano con diferentes dosis de aplicación de boro, del área experimental de CORBANA S.A. en finca La Rita, ubicada en la provincia de Limón, cantón de Pococí, distrito La Rita, durante el 2015. Las muestras fueron tomadas de la sección central interna de la tercera hoja (lámina) de cada planta, sin incluir la nervadura, así como en la sección central externa de la lámina (Figura 1).

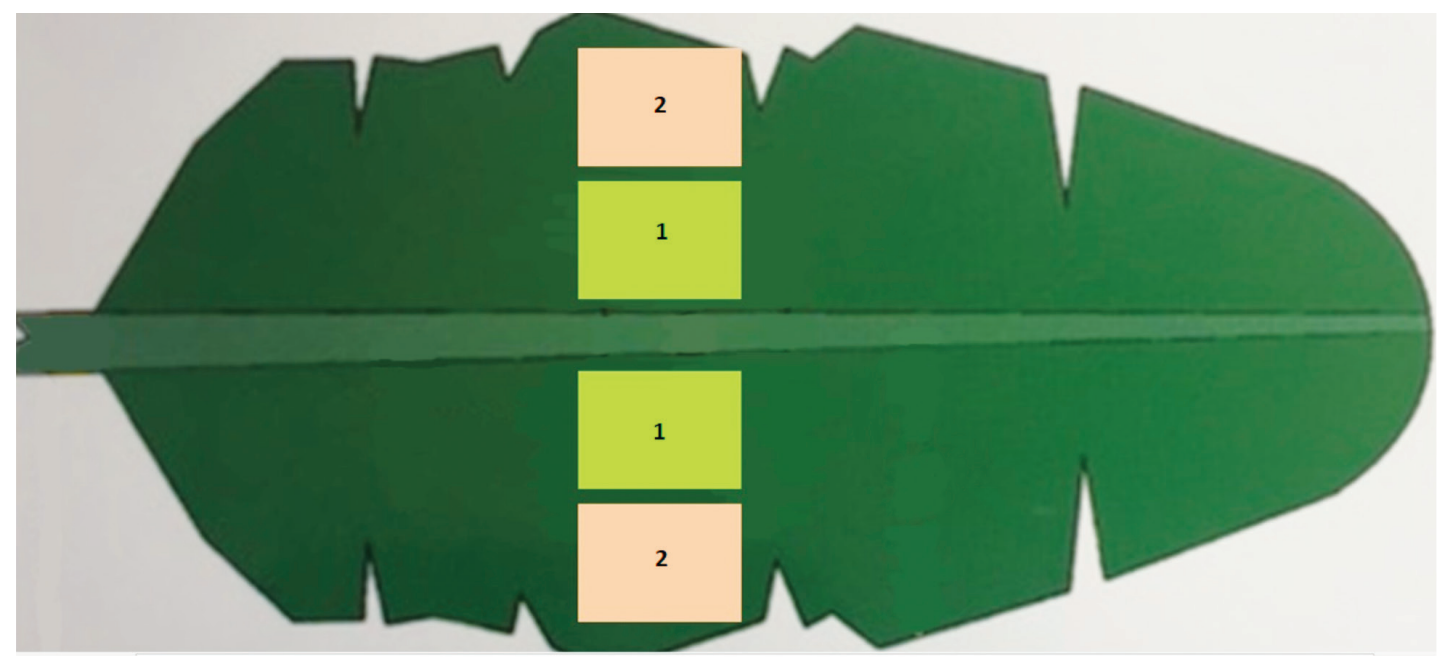

1. Sección central interna de la hoja 3

2. Sección central exterma de la hoja 3

Fig. 1. Método de muestreo foliar en la tercera hoja de la planta de banano (Musa AAA).

Las muestras de tejido foliar fueron lavadas con agua potable, secadas a $70^{\circ} \mathrm{C}$ por 3 días y molidas a un tamaño de partícula menor a 0,5 $\mathrm{mm}$. Se pesaron $0,5 \mathrm{gr}$ de muestra y fueron digeridos con $3 \mathrm{ml}$ de ácido nítrico y $3 \mathrm{ml}$ de peróxido de hidrógeno, en tubos de teflón, en un digestor de mircroondas Mars Xpress ${ }^{\circledR}$. Las muestras se calentaron por 10 minutos hasta alcanzar $210^{\circ} \mathrm{C}$ y se mantuvieron a esa temperatura durante 10 minutos adicionales. Posteriormente, se trasvasaron y diluyeron a $50 \mathrm{ml}$ con agua desionizada. El líquido resultante fue analizado en un equipo ICP/AES PerkinElmer Optima 7300 DV ${ }^{\circledR}$ a una longitud de onda de 249,676 nm. En este caso no se llevó a cabo el ajuste MSF (Multicomponent Spectral Fitting).

Se analizaron muestras de referencia de tejido foliar del programa International Plant Analytical-Exchange (IPE) de WEPAL, con diferentes contenidos de boro, con el fin de asegurar la exactitud del método analítico (WEPAL 2015b). Los datos de contenido de boro en suelo y foliar se analizaron mediante análisis de correlación y diagramas de frecuencia utilizando el programa Microsoft Excel 2013. 


\section{RESULTADOS}

\section{Disoluciones extractoras Mehlich 3 y fosfato de calcio}

A bajos niveles en suelo (menores a 0,2 mg..$^{-1}$ ) con la disolución Mehlich 3, se encontraron valores mayores, casi el doble de concentración en comparación con fosfato de calcio (Figura 2A). Conforme aumentó la concentración de boro, la correlación entre ambas disoluciones extractoras se incrementó. En la Figura 2B se observa que para niveles de 0,2 a $0,7 \mathrm{mg} \cdot \mathrm{l}^{-1}$ la correlación de los datos llegó a 68\%. En la Figura $2 \mathrm{C}$ se puede observar que a niveles de 0,7 a 2,0 mg. $1^{-1}$ la correlación aumentó a $95 \%$, por lo que se obtuvieron valores muy semejantes con ambas metodologías. Finalmente, en la Figura 2D se llega a una correlación de $98 \%$ entre ambas disoluciones extractoras, para niveles en suelo de 2,0 a $7,0 \mathrm{mg} \cdot \mathrm{l}^{-1}$.

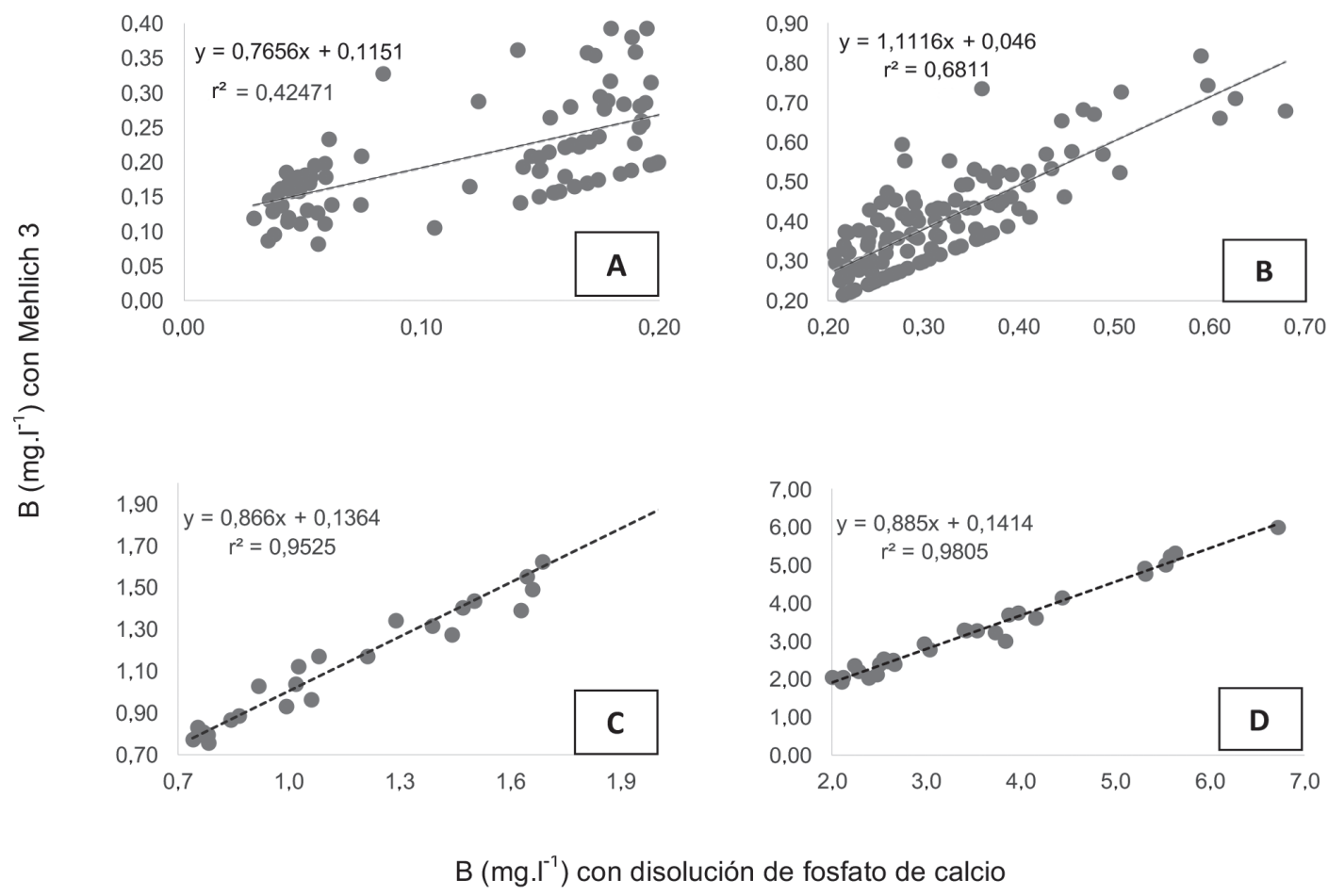

Fig. 2. Comparación de resultados de niveles de boro con la disolución Mehlich 3 vs la disolución extractora de fosfato de calcio en suelos con diferentes niveles de boro, A: menores a 0,2 mg.1 $1^{-1}$, B: 0,2 a 0,7 mg.1 ${ }^{-1}$, C: 0,7 a 2,0 mg. $1^{-1}$ y D: 2,0 a 7,0 mg. $\mathrm{l}^{-1}$.

En todos los casos, los niveles de boro encontrados, con la disolución extractora Mehlich 3 en las muestras de referencia (Cuadro 1), estuvieron comprendidos dentro los límites de aceptación al 95\% de confianza, determinados por WEPAL (2015a). Con base en ello, la determinación de boro en suelos de fincas bananeras se efectuó considerando dicho método. 
Cuadro 1. Niveles de boro en suelo (Mehlich 3) en muestras WEPAL, 2015.

\begin{tabular}{ccccc}
\hline Muestra & $\begin{array}{c}\text { Cantidad de laboratorios } \\
\text { que reportan }\end{array}$ & $\begin{array}{c}\mathrm{B}\left(\mathrm{mg} \cdot \mathrm{kg}^{-1}\right) \\
\text { Promedio general }\end{array}$ & $\begin{array}{c}\text { Límites de confianza } \\
\text { al 95\% }\end{array}$ & $\begin{array}{c}\mathrm{B}\left(\mathrm{mg}^{\mathrm{kg}} \mathrm{kg}^{-1}\right) \\
\text { Reportado por } \\
\text { CORBANA }\end{array}$ \\
\hline ISE 860 & 46 & 2,715 & $2,628-2,801$ & 2,670 \\
ISE 861 & 10 & 1,747 & $1,579-1,916$ & 1,890 \\
ISE 884 & 12 & 1,383 & $1,247-1,520$ & 1,280 \\
\hline
\end{tabular}

\section{Contenido de boro en suelos de fincas bananeras}

El valor promedio general de las fincas fue $0,58 \mathrm{mg} \cdot \mathrm{l}^{-1}$. El mayor valor encontrado fue $4,74 \mathrm{mg} . \mathrm{l}^{-1}$, el $90 \%$ de las muestras presentó niveles menores a $1,0 \mathrm{mg} . \mathrm{l}^{-1}$ (Cuadro 2). La mayoría de las muestras de suelo (64\%) presentaron niveles de 0,2 a $0,7 \mathrm{mg} . \mathrm{l}^{-1}$, un $11 \%$ mostraron niveles menores a 0,2 y un $24 \%$ concentraciones mayores al $0,7 \mathrm{mg} \cdot \mathrm{l}^{-1}$ (Figura 3).

Cuadro 2. Valores mínimos, máximos y promedio de boro encontrados en suelos de fincas bananeras mediante la disolución extractora Mehlich 3.

\begin{tabular}{|c|c|c|c|c|}
\hline \multirow[t]{2}{*}{ Finca } & \multicolumn{3}{|c|}{ Contenido de boro $\left(\mathrm{mg} . \mathrm{l}^{-1}\right.$ ) } & \multirow[t]{2}{*}{ Muestras por finca } \\
\hline & Mín. & Media & Máx. & \\
\hline 1 & 0,12 & 0,20 & 0,32 & 34 \\
\hline 2 & 0,10 & 0,20 & 0,38 & 22 \\
\hline 3 & 0,11 & 0,22 & 0,34 & 34 \\
\hline 4 & 0,13 & 0,22 & 0,31 & 56 \\
\hline 5 & 0,15 & 0,24 & 0,49 & 20 \\
\hline 6 & 0,18 & 0,31 & 0,52 & 29 \\
\hline 7 & 0,17 & 0,33 & 0,60 & 23 \\
\hline 8 & 0,20 & 0,34 & 0,73 & 24 \\
\hline 9 & 0,28 & 0,48 & 0,81 & 36 \\
\hline 10 & 0,20 & 0,50 & 0,70 & 24 \\
\hline 11 & 0,35 & 0,50 & 0,65 & 41 \\
\hline 12 & 0,36 & 0,51 & 0,65 & 14 \\
\hline 13 & 0,22 & 0,51 & 0,89 & 19 \\
\hline 14 & 0,40 & 0,61 & 0,88 & 26 \\
\hline 15 & 0,30 & 0,62 & 0,92 & 31 \\
\hline 16 & 0,38 & 0,68 & 1,36 & 30 \\
\hline 17 & 0,37 & 0,72 & 1,13 & 16 \\
\hline 18 & 0,36 & 0,77 & 1,18 & 29 \\
\hline 19 & 0,50 & 0,87 & 1,22 & 11 \\
\hline 20 & 0,62 & 1,00 & 1,74 & 33 \\
\hline 21 & 0,15 & 1,04 & 4,74 & 20 \\
\hline 22 & 0,57 & 1,11 & 2,73 & 31 \\
\hline 23 & 0,34 & 1,18 & 2,23 & 11 \\
\hline Promedio & 0,29 & 0,58 & 1,13 & Total: 614 \\
\hline
\end{tabular}




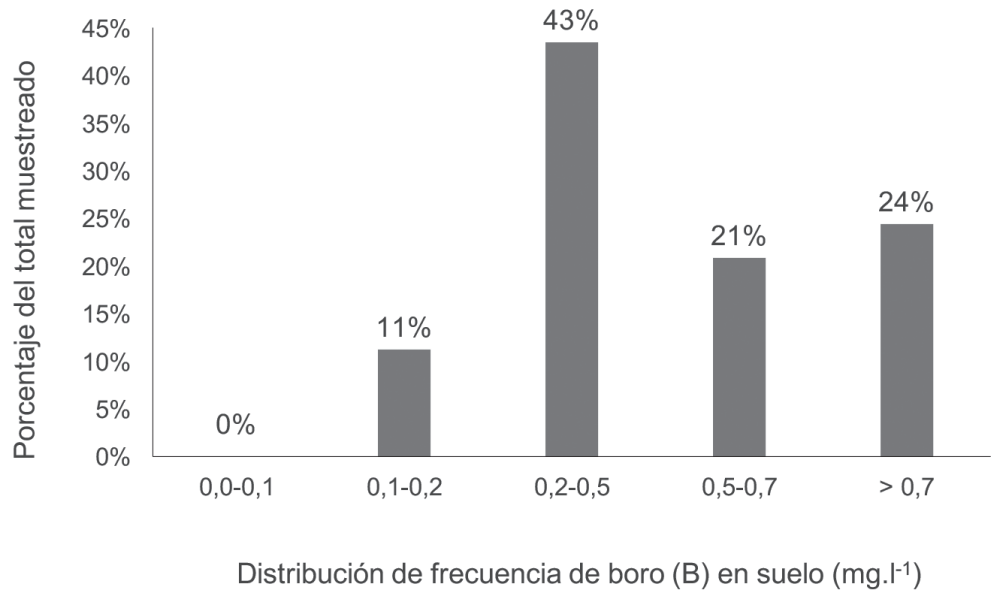

Fig. 3. Distribución de frecuencia de los niveles de boro en suelo con disolución extractora Mehlich 3 en fincas bananeras de Costa Rica.

\section{Contenido de boro en la planta de banano (tercera hoja)}

Los valores de boro en tejido foliar en la sección central interna de la tercera hoja, de plantas sembradas en suelos con contenidos de boro de 0,2 a $0,7 \mathrm{mg} \cdot \mathrm{l}^{-1}$, se presentaron en su mayoría en un ámbito de 10 a 15 mg.kg ${ }^{-1}$ en base seca (Figura 4). Cuando se extendió el ámbito de concentración en el suelo hasta 6,0 mg. $\mathrm{l}^{-1}$, los niveles foliares permanecieron en el ámbito de 10 a 15 mg.kg-1 para la sección central interna de la hoja (Figura 5A) y variaron de 10 hasta 70 mg.kg-1 , en la sección central externa (Figura 5B).

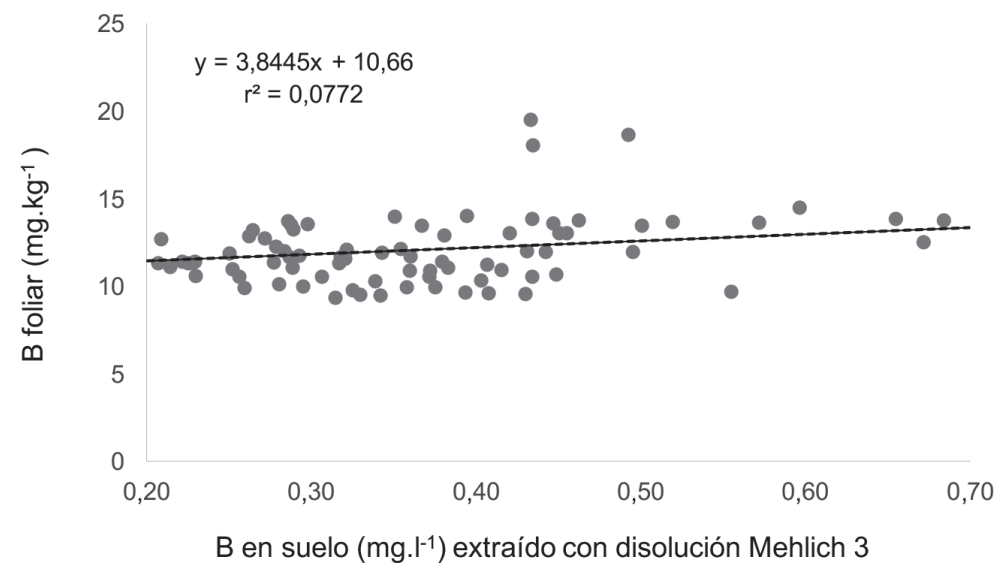

Fig. 4. Comparación de resultados de análisis de boro en suelo en niveles de 0,2 a $0,7 \mathrm{mg} . \mathrm{l}^{-1}$ contra los resultados de boro foliar en la sección central interna de la tercera hoja. 


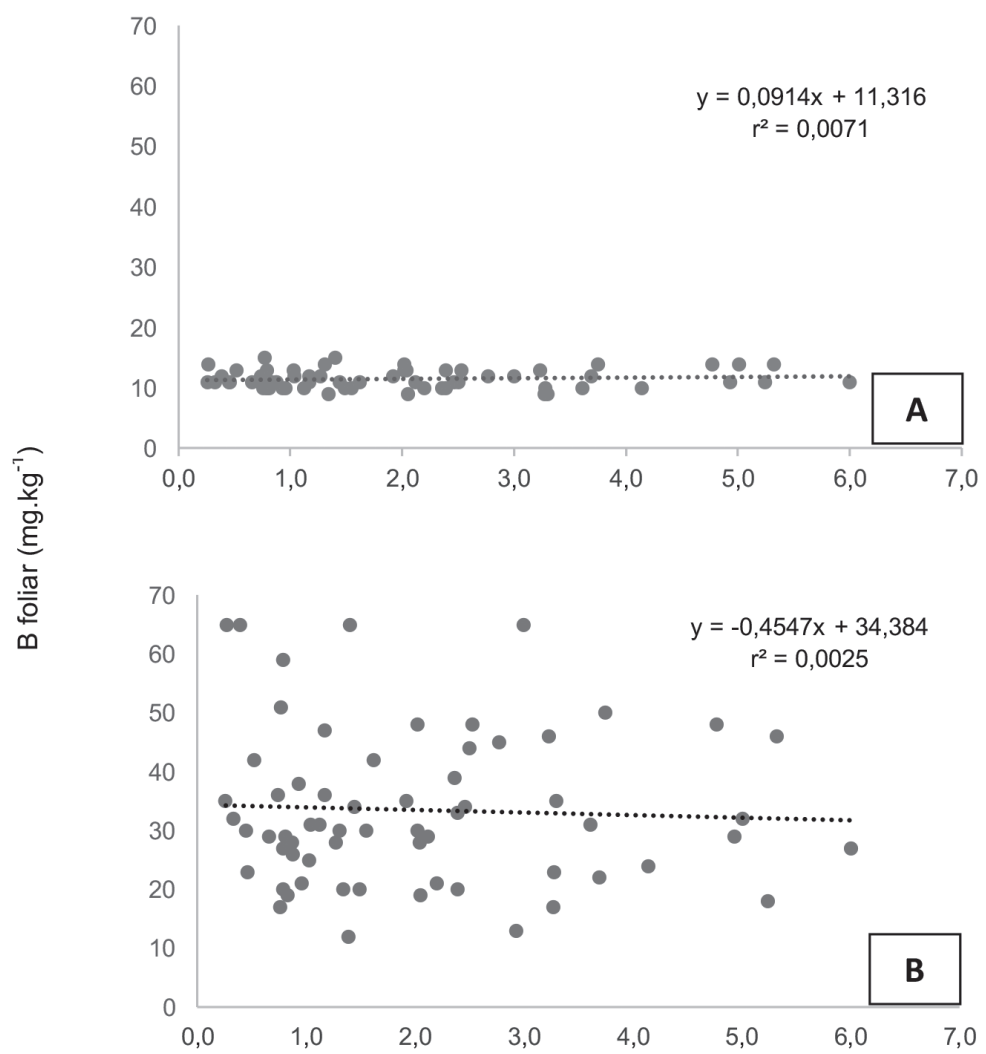

Fig. 5. Comparación de contenidos de boro en 2 secciones de la tercera hoja de plantas sembradas en suelo con niveles de boro de 0,2 a 6,0 mg.1 $1^{-1}$ A: sección central interna, B: sección central externa.

En todos los casos los niveles de boro foliar en las muestras de referencia IPE (Cuadro 3) estuvieron comprendidos dentro de los límites de aceptación al 95\% de confianza, determinados por WEPAL (2015b).

Cuadro 3. Niveles de Boro en muestras WEPAL de tejido foliar, 2015.

\begin{tabular}{ccccc}
\hline Muestra & $\begin{array}{c}\text { Cantidad } \\
\text { de laboratorios } \\
\text { que reportan }\end{array}$ & $\begin{array}{c}\text { B }\left(\mathrm{mg} \cdot \mathrm{kg}^{-1}\right) \\
\text { Promedio general }\end{array}$ & $\begin{array}{c}\text { Límites de confianza } \\
\text { al 95\% }\end{array}$ & $\begin{array}{c}\text { B }\left(\mathrm{mg}^{\mathrm{k}} \mathrm{kg}^{-1}\right) \\
\text { Reportado por } \\
\text { CORBANA }\end{array}$ \\
\hline IPE 177 & 73 & 48,03 & $47,11-48,96$ & 48,70 \\
IPE 214 & 90 & 18,86 & $18,33-19,40$ & 18,90 \\
IPE 169 & 65 & 19,74 & $19,30-20,18$ & 20,10 \\
\hline
\end{tabular}




\section{DISCUSIÓN}

\section{Disoluciones extractoras Mehlich 3 y fosfato de calcio}

La inclusión de boro como parte del análisis químico de suelos con la disolución extractora Mehlich 3, permitió contar con datos comparables a nivel internacional de una forma más rápida, confiable y con menos uso de reactivos químicos. Este tipo de extracción a niveles mayores a $0,2 \mathrm{mg} . \mathrm{l}^{-1}$ presenta una alta correlación con la obtenida mediante fosfato de calcio. La diferencia que se presenta entre ambas disoluciones extractoras a niveles bajos (menores a $0,2 \mathrm{mg} . \mathrm{l}^{-1}$ ) puede deberse a una mayor extracción con la disolución Mehlich 3, en comparación con el fosfato de calcio, debido a la mayor acidez de la primera. El $89 \%$ de los suelos bananeros evaluados mostró niveles de boro mayores a $0,2 \mathrm{mg} . \mathrm{l}^{-1}$, aspecto que facilita el uso de esta disolución como método de extracción de boro y, a la vez, permite la correlación con datos anteriores extraídos con fosfato de calcio. Estos resultados confirman la bondad del uso de la disolución Mehlich 3 para la determinación del contenido de boro en suelo tal como lo indican Schuman et al. (1992), Matula (2009) y Redd et al. (2008).

Es importante indicar que hubo necesidad de realizar un ajuste conocido como MSF (Multicomponent Spectral Fitting) en la lectura del equipo de plasma ICP-OES (PerkinElmer 1996) con el propósito de eliminar la interferencia de hierro determinada por Taber (2004) y Turner et al. (2016) en la lectura de boro a $249,676 \mathrm{~nm}$.

Al incluir el boro dentro de la rutina de extracción, para todas las muestras de suelo que son analizadas, se abre la oportunidad de nuevos estudios en esta área, para una correcta dosificación dentro de los programas de fertilización edáfica y foliar.

\section{Contenido de boro en suelos de fincas bananeras}

La mayoría de los suelos muestreados presentaron niveles adecuados de boro. Los niveles de boro en suelo recomendados para el cultivo de banano, según Coke y Boland (1971), citado por Ortega et al. (2009), deben encontrarse de 0,1 a 1,0 mg. $\mathrm{l}^{-1}$. El $89 \%$ de los suelos evaluados mostraron contenidos sobre el nivel crítico $(0,2$ $\mathrm{mg} .1^{-1}$ ) indicado en la Guía de Interpretación de análisis de suelo utilizada por el Centro Agronómico Tropical de Investigación y Enseñanza (CATIE) (Bertsch 1995) y sólo 10\% presentaron niveles mayores a 1,0 mg. $\mathrm{l}^{-1}$. Además, Ortega et al. (2009) encontraron que es a partir de niveles mayores a 2,08 mg..$^{-1}$ de boro en suelo que observaron síntomas de toxicidad y quema de bordes de las hojas. En el caso del boro, su deficiencia o toxicidad puede expresarse con mayor facilidad en comparación con el resto de los nutrimentos, dado que el ámbito entre deficiencia y toxicidad es muy pequeño (Vargas et al. 2007), debido a esto debe ser usado en forma muy cuidadosa.

\section{Contenido de boro en la planta de banano (tercera hoja)}

La metodología de determinación de boro a nivel foliar con digestión en microondas y lectura en ICP/OES es un método de utilización frecuente en muchos laboratorios del mundo, debido a su rapidez y alta precisión (WEPAL 2015b). Los ajustes que se utilizaron para el análisis de suelos no fueron requeridos a nivel foliar, debido al menor contenido de hierro en las hojas.

Los resultados obtenidos en la sección central interna de la tercera hoja coinciden con las observaciones de Vargas et al. (2007) quienes encontraron en plantas de banano asintomáticas, contenidos de boro de 10 a $15 \mathrm{mg} \cdot \mathrm{kg}^{-1}$. También coincide con Ortega et al. (2009), quienes determinaron el rango de 12 a 16 mg.kg-1 como el adecuado para el contenido de boro a nivel foliar. En la sección externa de las hojas los datos también 
coinciden con los reportados por Vargas et al. (2007) y Ortega et al. (2009), quienes encontraron que a mayores dosis de boro aplicado, los contenidos a nivel foliar en la sección externa de la hoja tienden a dispersarse significativamente, por lo que se encuentra acumulación de este elemento hacia el borde de la hoja. Esta acumulación, indica Marschner (1995), es el motivo por el cual ocurre una sintomatología de quema en los bordes de las hojas.

La inclusión de la determinación de boro, como parte del análisis químico de suelos con la disolución extractora Mehlich 3, permitió contar con datos comparables a nivel internacional de una forma más rápida y confiable. Esto es importante considerando la relevancia que dicho nutrimento tiene en la nutrición mineral de muchas de las plantas cultivables tropicales.

\section{LITERATURA CITADA}

Allen, SK; Jolley, VD; Webbs, BL; Shiffer, AK; Haby, VA. 2005. Challenges of Mehlich III Extraction for B and Comparison with other Methods in Boron-Treated Soils. In The ASA-CSSA-SSSA International Annual Meetings, 2005, Brigham Young University, Provo, Utah, EEUU. sp.

Arunkumar, BR; Thippeshappa, GN; Anjali, MC; Prashanth, KM. 2018. Boron: A critical micronutrient for crop growth and productivity. Journal of Pharmacognosy and Phytochemistry 7(2):2738-2741.

Berger, K; Troug, E. 1939. Boron Determination in Soils and Plants. Ind. Eng. Chem. Anal. Ed. 11 (10):540-545.

Bertsch, F. 1995. Manual para interpretar la fertilidad de los suelos de Costa Rica. Primera reimpresión. Editorial de la Universidad de Costa Rica. 81 p.

Brown, P; Bellaloui, M; Wimmer, M; Bassil, E; Ruiz, J; HU, H; Pfeffer, H; Daniel, F; Romheld, V. 2002. Boron in plant biology. Plant Biology 4:205-223.

Corrales, M; Bertsch, F; Bejarano, JA. 2005. Los laboratorios de análisis de suelos y foliares en Costa Rica: Informe del Comité de Laboratorios de Análisis de Suelos, Plantas y Aguas. Agronomía Costarricense 29(3):125-135.

Jeffrey, AJ; MacCallum, LE. 1988. Investigation of a hot $0.01 \mathrm{M} \mathrm{CaCl} 2$ soil boron extraction procedure followed by ICP-AES analysis. Commun. Soil Science Plant Analysis 19:663-673.

Marschner, H. 1995. Mineral Nutrition of Higher Plants. Segunda edición. London, UK, Academic 889 p.
Matula, J. 2009. Boron sorption in soils and its extractability by soil tests (Mehlich 3, ammonium acetate and water extraction). Plant Soil Environment 55(1):42-49.

Mehlich,A. 1984. Mehlich 3 soil test extractant: A modification of Mehlich 2 extractant. Communications in Soil Science and Plant Analysis 15(12):1409-1416.

Moreira, A; Castro, C; Fagenia, NK. 2011. Effects of Boron Application on Yield, Foliar Boron Concentration, and Efficiency of Soil Boron Extracting Solutions in a Xanthic Ferralsol Cultivated with Banana in Central Amazon. Communications in Soil Science and Plant Analysis 42(18):2169-2178.

Ortega, R; Serrano, E; Segura, R; Sandoval, J. 2009. Dosis crecientes de Boro (B) en el cultivo de banano (Musa AAA), In XVIII Congreso Latinoamericano de la Ciencia del Suelo, 2009, San José, Costa Rica. p. 1-11.

PerkinElmer. 1996. Interference Correction Windows. In Software Guide ICP-Emission Spectroscopy. The PerkinElmer Corporation, USA. p. 5-40.

Redd, SA; Shiffer, AK; Jolley, VD; Webbs, BL; Haby, VA. 2008. Mehlich 3 Extraction of Boron in Boron Treated Soils as Compared to Other Extractants. Communications in Soil Science and Plant Analysis 39(7-8):1245-1259.

Romeu-Díaz, R; Hunter, A. 1978. Metodología de muestreo de suelos, análisis químico de suelos y tejido vegetal y de investigaciones en invernadero. Centro Agronómico Tropical de Investigación y Enseñanza, Turrialba, Costa Rica. p. 78-62.

Schuman, LM; Bandel, VA; Donohue, SJ; Isaac, RA; Lippert, RM; Sims, JT; Tucker, MR. 1992. Comparison of Mehlich-1 and Mehlich -3 extractable soil boron with hot-water extractable boron. Communications in Soil Science and Plant Analysis 23(1-2):1-14.

Taber, HG. 2004. Boron Analysis of Mehlich No. 3 Extractant with Modified Inductive Coupled Argon Plasma Techniques to Eliminate Iron Interference. Communications in Soil Science and Plant Analysis 35(19-20):2957-2963.

Turner, BL; Bielnicka, A; Dalling, JW; Wolf, JA. 2016. Interference by Iron in the Determination of Boron by ICP-OES in Mehlich-III Extracts and Total Element Digests of Tropical Forest Soils. Communications in Soil Science and Plant Analysis. Doi: 10.1080/00103624.2016.1228952

Vargas, A; Arias, F; Serrano, E; Arias, O. 2007. Toxicidad de boro en plantaciones de banano (Musa AAA) en Costa Rica. Agronomía Costarricense 31(2):21-29.

Vargas, A. 1999. Síntomas de toxicidad y contenido de macro y micronutrientes en plantas de banano (Musa AAA) bajo condiciones de cultivo hidropónico. CORBANA 24(51):61-78. 
Vargas, A; Solis, P. 1999. Síntomas de deficiencia y contenido de macro y micronutrientes en plantas de banano (Musa AAA) bajo condiciones de carencia inducida en cultivo hidropónico. CORBANA 24(51):21-42.

WEPAL (Wageningen Evaluating Programs for Analytical Laboratories) 2015a. International Soil AnalyticalExchange Annual Report 2015. Wageningen University, Holanda. p. 207-209.
WEPAL (Wageningen Evaluating Programs for Analytical Laboratories) 2015b. International Plant AnalyticalExchange Annual Report 2015. Wageningen University, Holanda. p. 32-36.

Zbíral, J. 2016. Determination of plant-available micronutrients by the Mehlich 3 soil extractant - a proposal of critical values, Plant Soil Environment 62(11):527-531. 
